\title{
Letter \\ The key role of CD40 ligand in overcoming tumor-induced dendritic cell dysfunction
}

\author{
Alberto Pinzon-Charry ${ }^{1,2}$, Chris W Schmidt ${ }^{1,2}$ and José Alejandro López ${ }^{1,2}$
}

\begin{abstract}
${ }^{1}$ Dendritic Cell and Cancer Laboratory, Queensland Institute of Medical Research, Royal Brisbane Hospital Post Office, Brisbane 4019, Australia
${ }^{2}$ School of Medicine, University of Queensland, Herston 4006, Australia
\end{abstract}

Corresponding authors: José Alejandro, alej@@imr.edu.au; Alberto Pinzon-Charry, albertop@qimr.edu.au

Published: 22 February 2006

This article is online at http://breast-cancer-research.com/content/8/1/402

Breast Cancer Research 2006, 8:402 (doi:10.1186/bcr1386)

c) 2006 BioMed Central Ltd

See related commentary by Lenahan and Avigan [http://breast-cancer-research.com/content/8/1/101] and related research by Pinzon-Charry et al. [http://breast-cancer-research.com/content/8/1/R5] in this issue

\begin{abstract}
Overcoming dendritic cell (DC) dysfunction is a prerequisite for successful active immunotherapy against breast cancer. CD40 ligand (CD4OL), a key molecule in the interface between T-lymphocytes and DCs, seems to be instrumental in achieving that goal. Commenting on our data that CD40L protects circulating DCs from apoptosis induced by breast tumor products, Lenahan and Avigan highlighted the potential of CD40L for immunotherapy. We expand on that argument by pointing to additional findings that CD40L not only rescues genuine DCs but also functionally improves populations of immature antigen-presenting cells that fill the DC compartment in patients with breast cancer.
\end{abstract}

Crucial to the role of dendritic cells (DCs) as immune sentinels is their capacity to interact with lymphocytes [1]. This interaction mediates bi-directional signaling; DCs activate T lymphocytes (both CD4 and CD8) in an antigenspecific fashion and also receive signals from activated $T$ lymphocytes (CD4) via CD40L [2]. Indeed, the most efficient activation of cytotoxic CD8 lymphocytes required for tumor clearance seems to be the result of DC licensing after the interaction between CD40 and its ligand [3].

In this issue of Breast Cancer Research we show that patients with breast cancer exhibit a high rate of spontaneous apoptosis of circulating DCs [4]. Lenahan and Avigan [5] comment on our report, highlighting the protective function of IL-12 and CD40L against tumor-induced apoptosis. They suggest that the increased susceptibility of blood DCs to apoptosis might result in a diminished capacity of patients to respond to vaccines that depend on in vivo loading of DCs and recommend the use of DCs conditioned ex vivo to circumvent this problem. One alternative is the use of adjuvant cytokines or CD4OL to treat DCs. Indeed, in vitro stimulation with CD40L has been demonstrated to enhance the efficiency of DCs as antigen-presenting cells (APCs) for anti-tumoral immunization [6].

From an immunotherapy perspective such data are particularly relevant because DC-based therapies are being evaluated as vaccine adjuvants and are therefore being aggressively pursued in the treatment of a wide range of malignancies including breast cancer [7]. The design of DC vaccines to prevent the recurrence or achieve the regression of already existing tumors has been a major goal of immunotherapy. Blood DCs have been proposed for immunotherapy protocols because they offer the theoretical advantage of being in their natural state of differentiation and are thus capable of orchestrating immune responses in a more physiological manner.

Given that tumors are suppressive to DCs and induce significant alterations of the blood DC compartment, one must ask whether therapies using endogenous DCs can really enhance host immunity and delay or prevent tumor recurrence. In fact, immunotherapy protocols using circulating DC preparations have produced limited clinical responses [8]. Interestingly, it has been demonstrated that whereas circulating DCs isolated from patients with cancer are deficient in their function, DCs conditioned in vitro in the absence of tumor products have the capacity to induce robust immune responses [9,10]. We have therefore postulated that ex vivo conditioning of APCs should remain the most plausible approach in an effort to avoid tumorinduced suppression in vivo, and CD40 ligation may provide the optimal maturation signal to achieve this aim [11].

In addition, other CD40-CD40L interactions may have further effects on anti-tumor responses. For example, we have shown elsewhere that CD40 expression and engagement in 
a tumoral environment might be responsible for the accumulation of an immature (and dysfunctional) population within the lineage-negative HLA-DR-positive (commonly referred to as DCs) compartment in patients with breast cancer and other tumors [11]. We described these cells as $\mathrm{DR}^{+} \mathrm{ICs}$ (HLA-DR positive immature cells), having a deficient but recoverable antigen-presenting capacity. Interestingly, $\mathrm{DR}^{+} \mathrm{ICs}$ express higher levels of CD40 than genuine blood DCs, and are therefore potentially more responsive to the effects of CD40 ligation. We have proposed that the significant accumulation of $\mathrm{DR}^{+} \mathrm{ICs}$ in blood of these patients is the net result of tumor-induced apoptosis of DCs and resistance of immature APCs to apoptosis mediated by CD40-CD40L interactions. When measured in circulation, the number of $\mathrm{DR}^{+} \mathrm{IC}$ s often exceeds that of genuine blood DCs. Most importantly, we established that the antigenpresenting function in this population may be recovered by exposure to increased levels of CD40L in vitro [12].

If we accept that $e x$ vivo conditioning is required for effective immunotherapy, the next issue is to determine the optimal conditioning stimulus. As indicated above, immature APCs that are resistant to tumor-induced apoptosis and are thus highly abundant in patients with breast cancer are exquisitely sensitive to the effects of CD40 ligation [11]. Importantly, CD40L but no other maturation stimuli (inflammatory cytokines, CpG DNA, poly(l:C), lipopolysaccharide) induced both a vigorous activation of DCs and the robust functional maturation of immature APCs, as shown by the upregulation of the antigen-presenting machinery, the secretion of $\mathrm{IL}-12$ and enhanced T cell stimulatory capacities.

These data not only confirm that the help signal provided by CD40 engagement is one of the most potent stimuli for the maturation of DCs and other APCs (immature cells) that are abundant in cancer patients [11] but may also be important in preventing DC apoptosis induced by tumor products [4]. These findings advance $\mathrm{CD} 40 \mathrm{~L}$ as one of the prime candidates for the ex vivo conditioning of cellular vectors for immunotherapy.

\section{Competing interests}

The authors declare that they have no competing interests.

\section{References}

1 Banchereau J, Briere F, Caux C, Davoust J, Lebecque S, Liu YJ, Pulendran B, Palucka K: Immunobiology of dendritic cells. Annu Rev Immunol 2000, 18:767-811.

2 Schoenberger SP, Toes RE, van der Voort El, Offringa R, Melief CJ: T-cell help for cytotoxic $T$ lymphocytes is mediated by CD40-CD40L interactions. Nature 1998, 393:480-483.

3 Ridge JP, Di Rosa F, Matzinger P: A conditioned dendritic cell can be a temporal bridge between a $\mathrm{CD} 4^{+} \mathrm{T}$-helper and a Tkiller cell. Nature 1998, 393:474-478.

4 Pinzon-Charry A, Maxwell T, McGuckin MA, Schmidt C, Furnival C, Lopez JA: Spontaneous apoptosis of blood dendritic cells in patients with breast cancer. Breast Cancer Res 2006, 8:R5.

5 Lenahan C, Avigan D: Dendritic cell defects in patients with cancer: mechanisms and significance. Breast Cancer Res 2006, 8:101.
6 Terheyden P, Straten P, Brocker EB, Kampgen E, Becker JC: CD40-ligated dendritic cells effectively expand melanomaspecific CD8+ CTLs and CD4+ IFN- $\gamma$-producing T cells from tumor-infiltrating lymphocytes. J Immunol 2000, 164:66336639.

7 Lopez JA, Hart DN: Current issues in dendritic cell cancer immunotherapy. Curr Opin Mol Ther 2002, 4:54-63.

8 Figdor CG, de Vries IJ, Lesterhuis WJ, Melief CJ: Dendritic cell immunotherapy: mapping the way. Nat Med 2004, 10:475480.

9 Ratta M, Fagnoni F, Curti A, Vescovini R, Sansoni P, Oliviero B, Fogli M, Ferri E, Della Cuna GR, Tura S, et al.: Dendritic cells are functionally defective in multiple myeloma: the role of interleukin-6. Blood 2002, 100:230-237.

10 Gabrilovich DI, Corak J, Ciernik IF, Kavanaugh D, Carbone DP: Decreased antigen presentation by dendritic cells in patients with breast cancer. Clin Cancer Res 1997, 3:483-490.

11 Pinzon-Charry A, Ho C, Laherty R, Maxwell T, Walker D, Gardiner $\mathrm{R}$, O'Connor L, Pyke C, Schmidt CW, Furnival C, et al:: A population of HLA-DR+ immature cells accumulate in the blood dendritic cell compartment of patients with different types of cancer. Neoplasia 2005, 7:1123-1132.

12 Pinzon-Charry A, Maxwell T, Prato S, Furnival C, Schmidt CW, Lopez JA: HLA-DR+ immature cells exhibit reduced antigen presenting cell function but respond to CD40 stimulation. Neoplasia 2005, 7:1112-1122. 